\title{
Horizontal Acoustic Barriers for Protection from Seismic Waves
}

\author{
Sergey V. Kuznetsov ${ }^{1}$ and Aybek E. Nafasov ${ }^{2}$ \\ ${ }^{1}$ Institute for Problems in Mechanics, Russian Academy of Sciences, Moscow 119526, Russia \\ ${ }^{2}$ Department Strength of Materials, Moscow State University of Civil Engineering, Moscow 129337, Russia
}

Correspondence should be addressed to Sergey V. Kuznetsov, kuzn-sergey@yandex.ru

Received 7 May 2011; Accepted 10 June 2011

Academic Editor: Emil Manoach

Copyright ( $\odot 2011$ S. V. Kuznetsov and A. E. Nafasov. This is an open access article distributed under the Creative Commons Attribution License, which permits unrestricted use, distribution, and reproduction in any medium, provided the original work is properly cited.

The basic idea of a seismic barrier is to protect an area occupied by a building or a group of buildings from seismic waves. Depending on nature of seismic waves that are most probable in a specific region, different kinds of seismic barriers can be suggested. Herein, we consider a kind of a seismic barrier that represents a relatively thin surface layer that prevents surface seismic waves from propagating. The ideas for these barriers are based on one Chadwick's result concerning nonpropagation condition for Rayleigh waves in a clamped half-space, and Love's theorem that describes condition of nonexistence for Love waves. The numerical simulations reveal that to be effective the length of the horizontal barriers should be comparable to the typical wavelength.

\section{Introduction}

1.1. Methods of Seismic Protection. Generally, current methods for preventing failure of structures due to seismic activity can be divided into two groups: (i) approaches for creating seismically stable structures and joints; this group contains different methods ensuring either active or passive protection; (ii) approaches for creating a kind of seismic barrier preventing seismic waves from transmitting wave energy into a protected region.

While the first group includes a lot of different engineering approaches and solutions, the second one contains very few studies; see Takahashi et al. [1] and more recent works by Motamed et al. [2], Kusakabe et al. [3], and Kuznetsov [4]. The current research belongs to the second group.

1.2. Possible Types of Wave Barriers. The considered seismic barriers can be of two types: vertical, aimed to reflect, trap, and dissipate most of the seismic wave energy; horizontal, based on Chadwick and Smith [5] and Love [6] theorems and aimed to prevent certain types of seismic waves from propagation, see (Figure 1).

Yet another interesting approach is to create a "rough" surface of the half-space to force the propagating Rayleigh wave scatter by caves and swellings, see (Figure 2), where part of a free surface with the sinusoidal roughness is pictured. In this respect, the rough surface apparently transforms the elastic half-space into viscoelastic one. To be effective, periodic imperfections should have magnitude and period comparable to the magnitude and wavelength of propagating Rayleigh wave [7-9].

In practice, such a rough surface can be achieved by a series of rather deep trenches oriented transversally to the most probable direction of the wave front. Some of the obvious deficiencies of this method are (i) its inability to persist the surface waves other than Rayleigh waves; (ii) protection from Rayleigh waves travelling only in directions that are almost orthogonal to orientation of the trenches; (iii) high sensitivity to the frequency of travelling Rayleigh waves. These shortcomings made an idea of exploiting a rough surface as a kind of protective barrier unrealizable.

1.3. Horizontal Barriers. Horizontal barriers can be constructed by modifying properties of the outer layer preventing the corresponding surface wave from propagation.

In practice, modifying physical properties of the outer layer can be achieved by reinforcing ground with piles or 
"soil nails"; see papers where reinforcing was studied in a context of increasing bearing load of soil [10-14].

If distance between piles is sufficiently smaller than the wave length, then a reinforced region can be considered as macroscopically homogeneous and either transversely isotropic or orthotropic depending on the arrangement of piles. Naturally, the homogenized physical properties of the reinforced medium will depend upon the material of piles, distance between them, and their arrangement.

For stochastically homogeneous arrangement of piles and the initially isotropic upper soil layer, the reinforced soil layer becomes transversely isotropic with the homogenized (effective) characteristics that can be evaluated by different methods.

Voigt Homogenization. Yields the upper bound for effective characteristics [15]

$$
\mathbf{C}_{\text {effective }}=(1-f) \mathbf{C}_{\text {soil }}+f \mathbf{C}_{\text {piles }} \text {, }
$$

where $\mathbf{C}_{*}$ are the corresponding elasticity tensors, and $f$ is the average volume fraction of piles.

Reuss Homogenization. This method is related to constructing the homogenized inverse tensors

$$
\mathbf{S}_{\text {effective }}=(1-f) \mathbf{S}_{\text {soil }}+f \mathbf{S}_{\text {piles }}
$$

it yields the lower bound, where $\boldsymbol{S}_{*}$ are the corresponding compliance tensors. In the case of pile reinforcement, these two methods give too broad "fork" and, thus, lead to a nonreliable estimate.

Two-Scale Asymptotic Expansion Method. Much more accurate results yield the two-scale asymptotic expansion method $[15,16]$

$$
\mathbf{C}_{\text {effective }}=(1-f) \mathbf{C}_{\text {soil }}+f \mathbf{C}_{\text {piles }}+\mathbf{K} \text {, }
$$

where $\mathbf{K}$ is the corrector that is defined by solving the special boundary value problem for a typical translational invariant cell. It is interesting to note that taking the corrector $\mathbf{K}$ in (3) as the null tensor, we arrive at Voigt homogenization (1).

Methods for constructing the corrector within the twoscale asymptotic expansion methods are discussed by Michel et al. [17], Cecchi and Rizzi [18].

\section{The Main Types of Surface Acoustic Waves}

In this section, we proceed to the analyzes of the main types of seismic surface waves and conditions for their nonexistence.

2.1. Rayleigh Waves. These waves discovered by Lord Rayleigh [19] propagate on a plane surface of a half-space; see, (Figure 3) and exponentially attenuate with depth. These waves transmit the most seismic energy and lead to most severe damage in earthquakes.

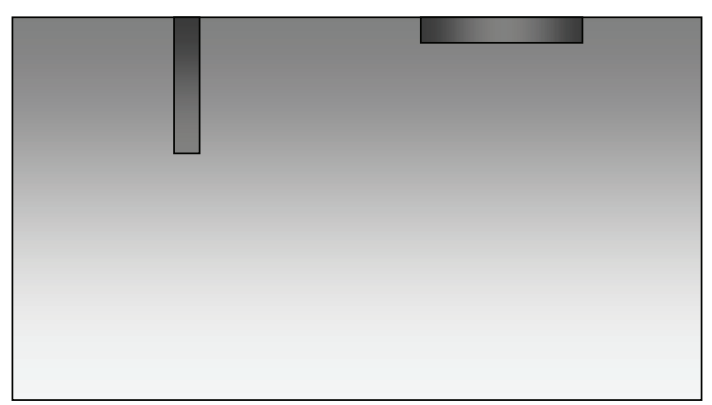

Figure 1: Vertical and horizontal seismic barriers.

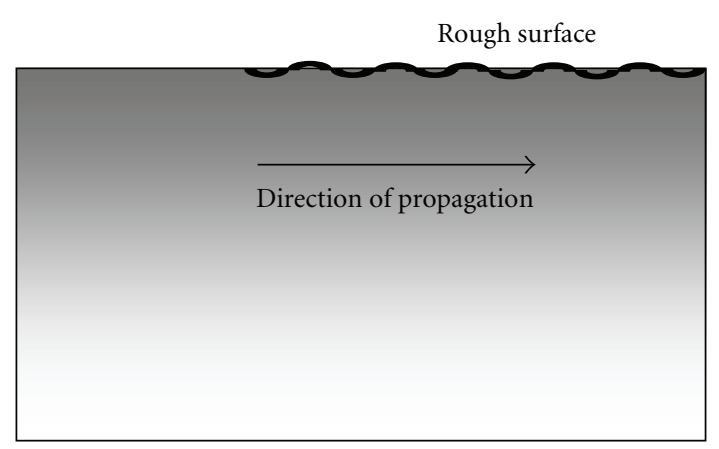

FIGURE 2: Rough surface acting as a seismic barrier against Rayleigh waves.

One interesting problem associated with Rayleigh waves is the search of "forbidden" directions of "forbidden" (necessary anisotropic) materials that does not transmit a Rayleigh wave along some directions. Forbidden materials and forbidden directions have been intensively searched both experimentally and numerically [20-22] until midseventies when the theorem of existence for Rayleigh waves was rigorously proved $[5,23-28]$. This theorem states that no materials possessing forbidden directions for Rayleigh waves can exist.

Despite proof of the theorem of existence, a small chance for existence of forbidden materials remained. This corresponded to the case of nonsemisimple degeneracy of a special matrix associated with the first-order equation of motion; actually, this matrix is the Jacobian for the Hamiltonian formalism used for Rayleigh wave description. However, it was shown [29] that even at the non-semisimple degeneracy a wave resembling the genuine Rayleigh wave can propagated. Thus, for waves propagating on a homogeneous half-space, no forbidden materials or directions can exist.

2.2. Stoneley Waves. These waves were introduced by Stoneley [30] and analyzed in[31-33]. Stoneley waves propagate on an interface between two contacting half-spaces, (Figure 4).

In contrast to Rayleigh waves, Stoneley waves can propagate only if material constants of the contacting half-spaces satisfy special (very restrictive) conditions of existence. These conditions were studied by Chadwick and Borejko [34], Sengupta and Nath [35]. 


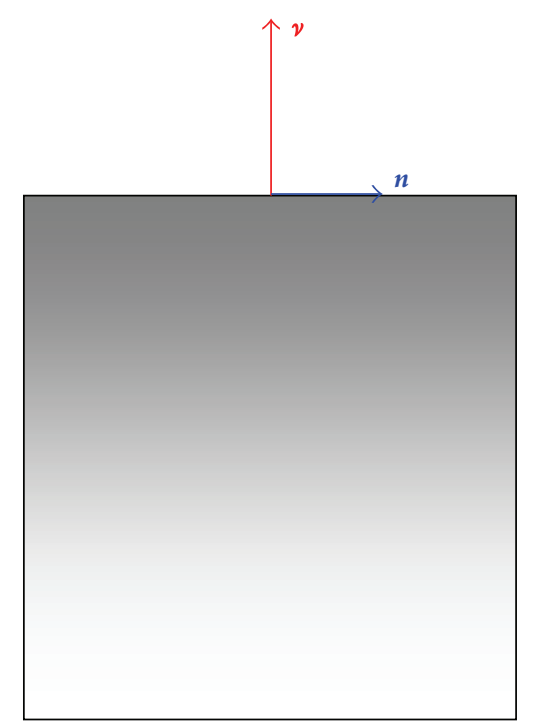

FIgURE 3: Rayleigh wave in a half-space.

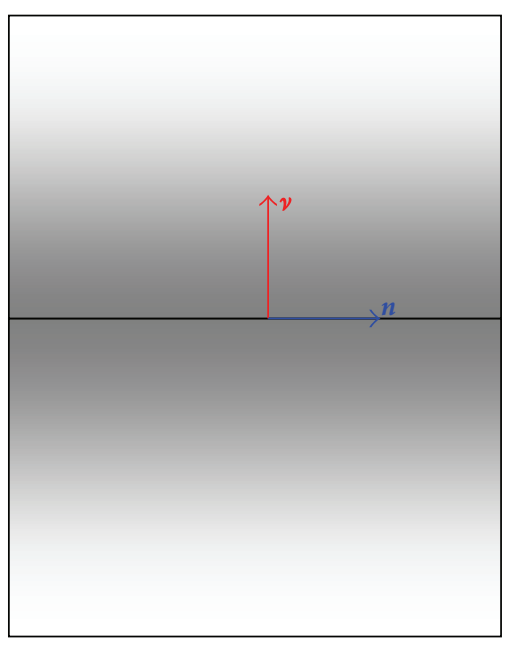

FIGURE 4: Stoneley wave on the interface between two contacting half-spaces.

It should be noted that for the arbitrary anisotropy no closed analytical relations between material constants of the contacting half-spaces ensuring existence or nonexistence of Stoneley waves have been found (2010).

2.3. Love and $\mathrm{SH}$ Waves. Love waves [6] are horizontally polarized shear waves that propagate on the interface between an elastic layer contacting with elastic half-space, (Figure 7). At the outer surface of the layer, traction-free boundary conditions are generally considered.

In the case of both isotropic layer and half-space, the conditions of existence derived by Love are

$$
c_{\text {layer }}^{S}<c_{\text {halfspace }}^{S},
$$

where $c_{*}^{S}$ are the corresponding speeds of the transverse bulk waves. At violating condition (4), no Love wave can

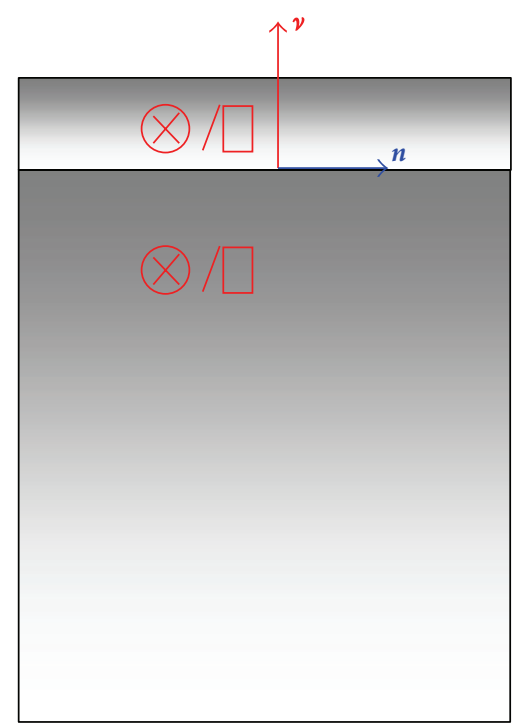

Figure 5: Love wave propagating on the interface.

propagate. For the case of both anisotropic (monoclinic) layer and a half-space, the condition of existence is also known [36].

$\mathrm{SH}$ waves resemble Love waves in polarization but differ in the absence of the contacting half-space. At the outer surfaces of the layered plate, different boundary conditions can be formulated [37]. In contrast to genuine Love waves, the $\mathrm{SH}$ waves exist at any combination of elastic properties of the contacting layers.

Besides Love and $\mathrm{SH}$ waves, a combination of them can also be considered. This corresponds to a horizontally polarized wave propagating in a layered system consisting of multiple layers contacting with a half-space. Analysis of conditions of propagation for such a system can be done by applying either transfer matrix method [38, 39], known also as the Thomson-Haskell method due to its originators, or the global matrix method mainly developed by Knopoff [40].

At present (2010), no closed analytical conditions of existence for the combined Love and $\mathrm{SH}$ waves propagating in anisotropic multilayered systems are known; however, these conditions can be obtained numerically by applying transfer or global matrix methods; see [36, 37, 41].

Different observations show that genuine Love and the combined Love-SH waves along with Rayleigh and RayleighLamb waves play the most important role in transforming seismic energy in earthquakes (e.g., $[42,43])$. But, as we have seen, there is a relatively simple (at least from a theoretical point of view) method for stopping Love and the combined Love and SH waves by modifying the outer layer in such a way that conditions of existence (4) are violated.

2.4. Lamb and Rayleigh-Lamb Waves. Lamb waves [44] are dispersive waves propagating in a homogeneous plate and (if a plate is isotropic) polarized in the sagittal plane, similarly to polarization of the genuine Rayleigh waves. It is known $[45,46]$ that Lamb waves can propagate at any anisotropy of 


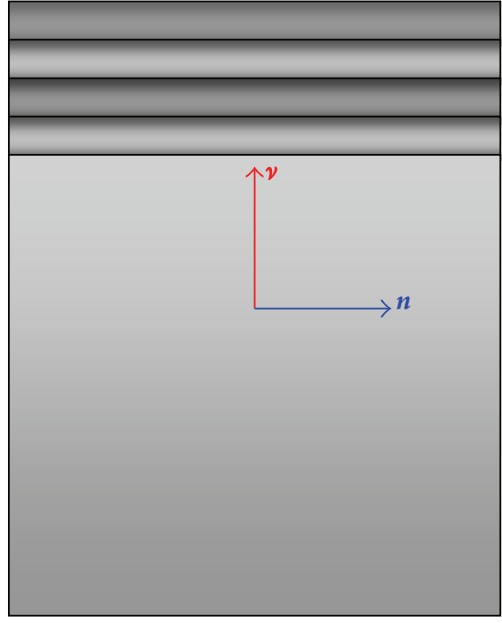

Figure 6: Rayleigh-Lamb waves.

the layer and at traction-free, clamped, or mixed boundary conditions imposed on the outer surfaces of the plate. The same result can be extrapolated to a layered plate containing multiple anisotropic homogeneous layers in a contact [47]. Thus, for Lamb waves, no forbidden materials exist.

More interesting from seismological point of view are Rayleigh-Lamb waves; see (Figure 6). These are dispersive waves propagating in a layered plate contacting with a (homogeneous) half-space. Rayleigh-Lamb waves in isotropic media are polarized in the sagittal plane defined by vectors $v$ (normal to a median plane) and $\mathbf{n}$ (direction of propagation), as Lamb and Rayleigh waves. Needless to say that Rayleigh-Lamb waves are much more difficult for theoretical studies than Rayleigh or Lamb waves.

\section{Horizontal Seismic Barriers}

Herein, we present some results on numerical simulation of propagating seismic waves and their interaction with horizontal seismic barriers. The presented results were obtained by the explicit FE code.

3.1. Basic Observations. Our analyses revealed that similarly to vertical barriers, the transverse barriers should satisfy several conditions to effectively protect from seismic waves: (i) length (horizontal) of the barrier should be comparable to the wavelength; (ii) material of the barrier should have larger density than the ambient soil for Rayleigh waves; that is, in agreement with Chadwick's theorem stating that at the clamped surface of a halfspace, no Rayleigh wave can propagate; (iii) material of the barrier should satisfy the opposite Love's propagating condition (4) for protecting from propagating seismic Love waves.

Figure 7 demonstrates a movie frame related to numerical simulation of a propagating seismic Rayleigh wave having long wavelength and interacting with a roundshaped transverse (horizontal) barrier; the latter completely surrounds the protected region. The ratio of the wavelength to the length of the barrier was taken one and a half.
Inside the protected region, reduction of the magnitude of displacements was about three times comparing to magnitude of displacements at the outside territory.

3.2. $2 \mathrm{D} \mathrm{Model.} \mathrm{To} \mathrm{simplify} \mathrm{the} \mathrm{subsequent} \mathrm{analyses,} \mathrm{a} 2 \mathrm{D}$ model was used for numerical studies; (Figure 8).

In the center of the upper edge of a plate a harmonic loading was applied allowing us to model propagation of seismic wave. To analyze effectiveness of the barrier, the magnitude of displacements was evaluated at two points on the upper edge located at equal distances from the epicenter: one point in the left part of the plate and another in the right part behind the barrier.

Comparing both sides of a plate gives information on the effectiveness of the barrier in respect of the reduction of the magnitude in the right side of a plate behind the barrier.

3.3. Dimensional Analysis. In accordance with Buckingham's pi-theorem [48-50] stating that a physical law is independent of the form of the units, the displacement field $\mathbf{u}$ in a reference point $\mathbf{x}_{0}$ behind the barrier can be described by the following set of dimensionless parameters

$$
\mathbf{u}\left(\frac{\mathbf{x}_{0}}{\lambda_{1}} ; \nu_{1}, \nu_{2} ; \frac{E_{1}}{E_{2}} ; \frac{\rho_{1}}{\rho_{2}} ; \frac{L}{\lambda_{1}} ; \frac{D}{\lambda_{1}} ; \frac{\omega \lambda_{1}}{\sqrt{E_{1} / \rho_{1}}}\right),
$$

where index 1 the ambient material of the half-space, index 2 is the barrier; $\lambda_{1}$ is the wavelength (of a longitudinal bulk wave in a half-space); $v_{k}$ are the corresponding Poisson's ratios; $E_{k}$ are Young's modules; $\rho_{k}$ are densities; $L$ and $D$ are geometrical dimensions of the barrier; $\omega$ is the wave circular frequency.

The preliminary analysis revealed that both Poisson's ratios have almost no influence on the displacements at $\mathbf{x}_{0}$. Thus, eliminating both Poisson's ratios, and assuming that the wave frequency remains constant (that also implies the constant wavelength), the expression (5) can be simplified as follows:

$$
\mathbf{u}\left(\frac{E_{1}}{E_{2}} ; \frac{\rho_{1}}{\rho_{2}} ; \frac{L}{\lambda_{1}} ; \frac{D}{\lambda_{1}}\right)
$$

3.4. Variation of $E_{1} / E_{2}$. Variation of the magnitude of displacements at the reference point $\mathbf{x}_{0}$ due to variation of ratio $E_{1} / E_{2}$ and at fixed other parameters in (6) is presented in (Figure 10).

The charts in Figure 10 are plotted at $\nu_{1}=\nu_{2}=$ $0.25 ; \rho_{1}=\rho_{2}=1 ; \omega=0.1 ; L=25 ; D=1$.

The most interesting and surprising deduction that can be made from analyzing these charts states that a relatively broad variation of Young's modulus of the barrier at the constant modulus of the half-space almost negligibly influences magnitude of displacements in the protected zone.

3.5. Variation of $\rho_{1} / \rho_{2}$. Variation of the magnitude of displacements at the reference point $\mathbf{x}_{0}$ due to variation of ratio $\rho_{1} / \rho_{2}$ and at fixed other parameters in (6), is presented in (Figure 11). 


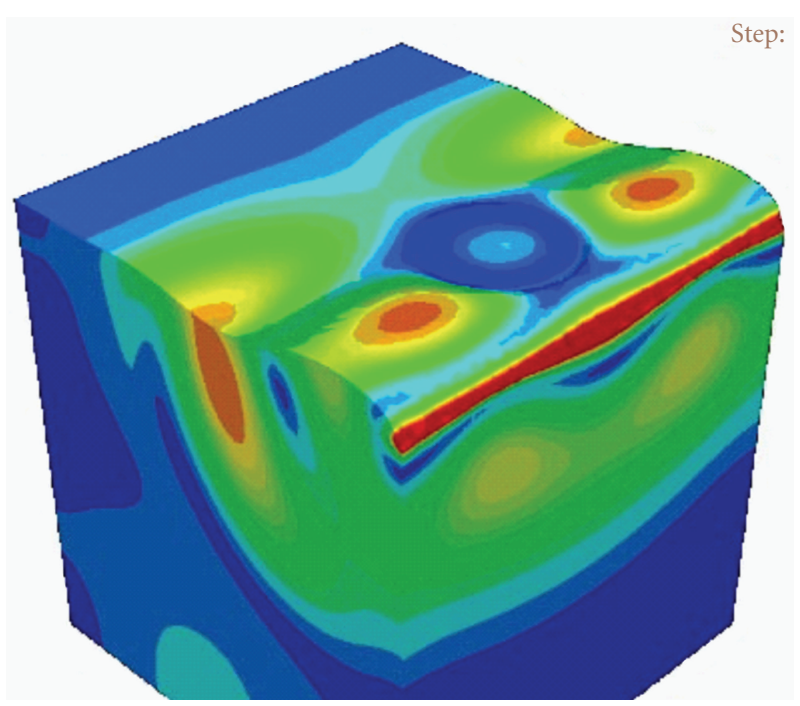

(a)

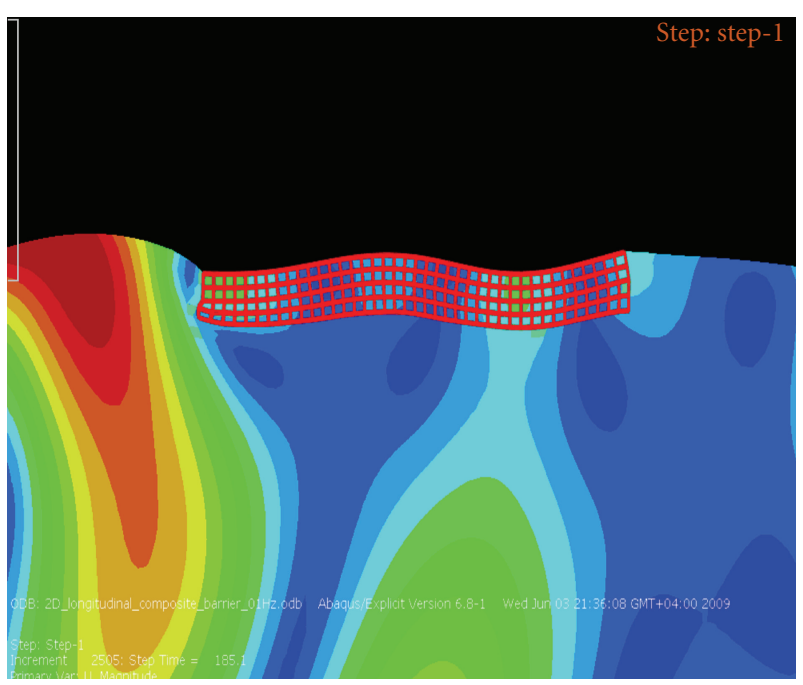

(b)

FIGURE 7: 3D model of the horizontal round-shaped barrier interacting with a long Rayleigh wave: (a) 3D model; (b) cross-section near the barrier.

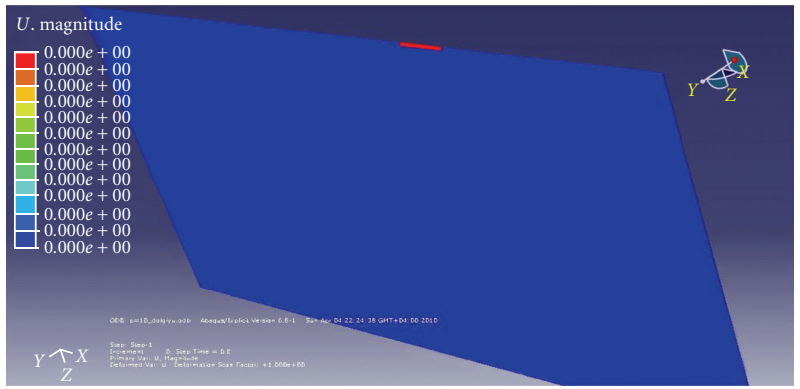

(a)

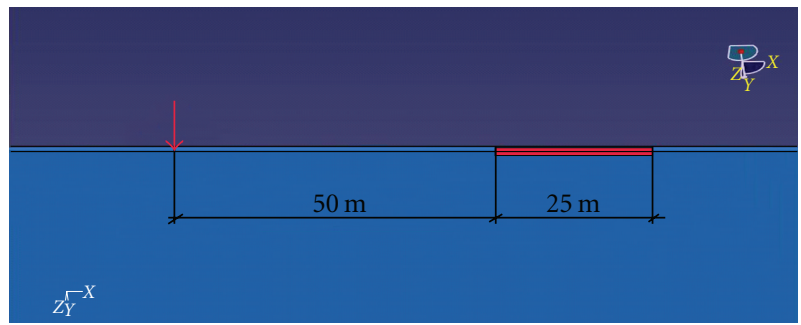

(b)

FIGURE 8: 2D model with seismic barrier.

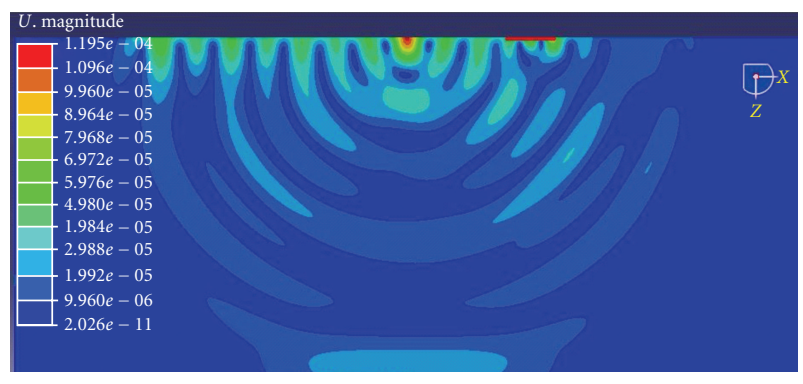

FIGURE 9: Seismic waves emanating from the epicenter.

The charts in (Figure 11) are plotted at $\nu_{1}=v_{2}=$ $0.25 ; E_{1}=E_{2}=1 ; \omega=0.1 ; L=25 ; D=1$. Now, the obtained data reveal that the displacements behind the barrier can be considerably reduced at decreasing ratio $\rho_{1} / \rho_{2}$. Thus, to be the most efficient, the barrier should be made of a more dense material than the ambient soil.

3.6. Varying Geometry of the Barrier. Herein, the influence of the relative dimensions of the barrier $L / \lambda_{1}$ and $D / \lambda_{1}$ on the magnitude of displacements in a reference point behind the barrier is analyzed.

Figure 12 demonstrates how increasing the relative length $L / \lambda_{1}$ of the barrier reduces the magnitude of displacements.

Similarly, the dependence of the magnitude of displacements on the depth of the barrier is given on (Figure 13).

As can be predictable, increasing either relative length or relative depth of the barrier can considerably reduce magnitude of vibrations in the protected zone.

\section{Concluding Remarks}

Herein, a brief outline of future research directions related to creating more efficient seismic barriers is given. A practically important case, when seismic barriers appear to be indispensable, is discussed in the last subsection.

4.1. Settingup an Optimization Problem. To make search of the optimal geometric and physical properties of the protecting barriers more systematic, solution of the following optimizing problem can be suggested. Mathematically, the 


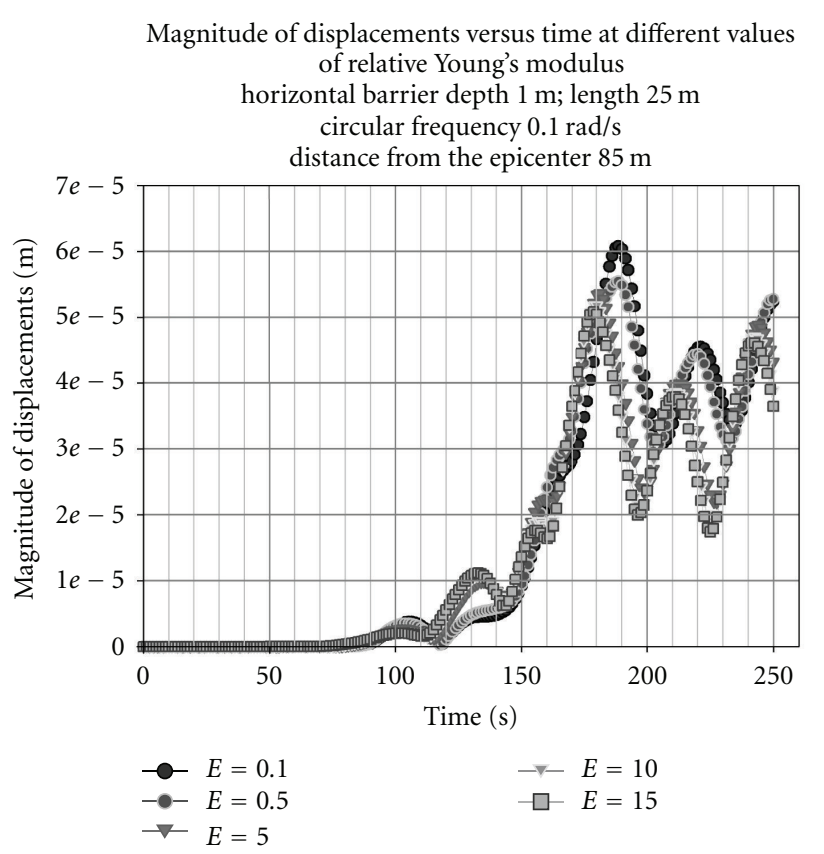

Figure 10: Variation of the magnitude of displacements at a fixed point behind the barrier due to variation of $E_{1} / E_{2}$.

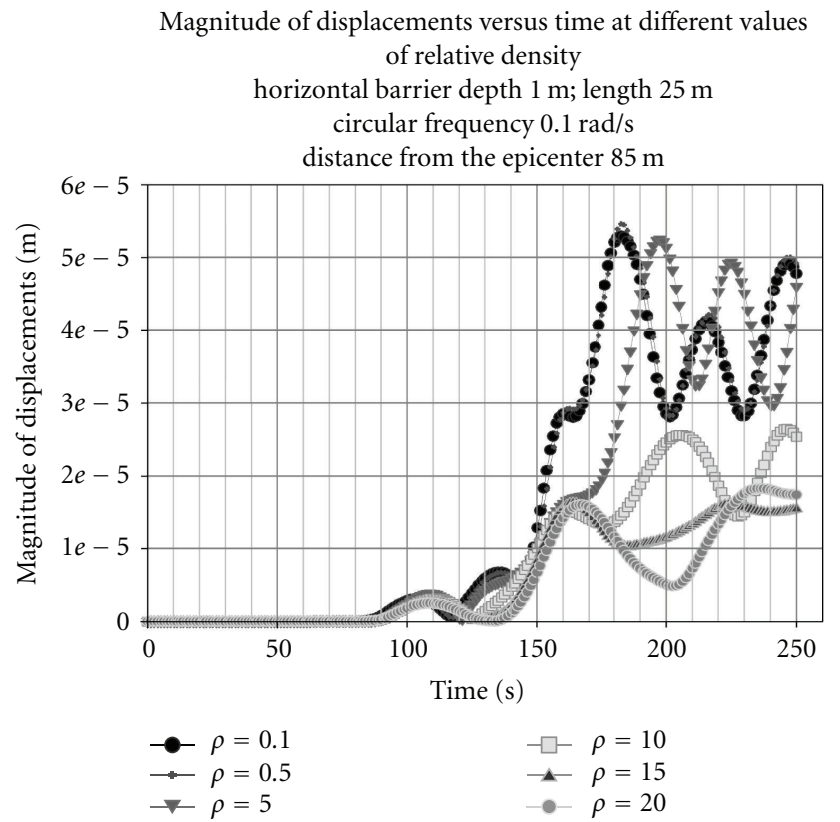

Figure 11: Variation of the magnitude of displacements at a fixed point behind the barrier due to variation of $\rho_{1} / \rho_{2}$.

optimization problem for minimizing magnitudes of deflections can be written as finding minimum of the following target function $F$ :

$$
\min _{\mathbf{C}_{1}, \rho_{1}, l_{1}, h_{1}}\left(F\left(\mathbf{C}_{1} ; \rho_{1} ; l_{1} ; h_{1}\right) \equiv\left(\max _{\omega \in \Omega} \max _{x \in D}[s(\omega) m(x, \omega)]\right)\right),
$$

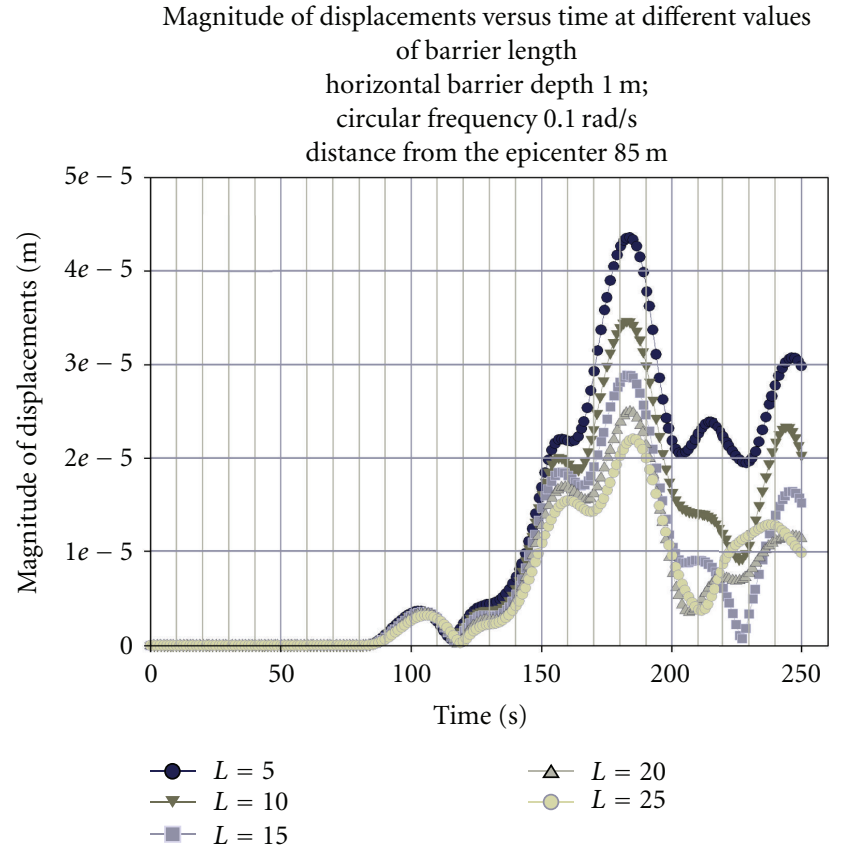

FIGURE 12: Variation of the magnitude of displacements at a fixed point behind the barrier due to variation of the relative length $L / \lambda_{1}$.

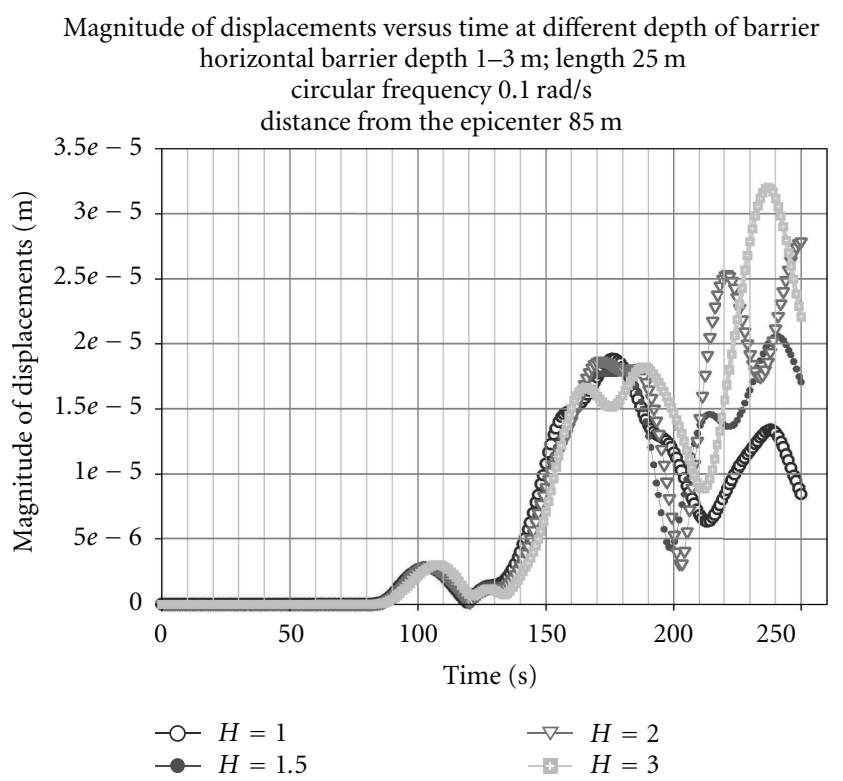

FIGURE 13: Variation of the magnitude of displacements at a fixed point behind the barrier due to variation of the relative depth $D / \lambda_{1}$.

where $\mathbf{C}_{1}, \rho_{1}, h_{1}$, and $l_{1}$ are the elasticity tensor, density, depth, and length of the barrier (in the case of isotropic material, Lamé constants can be used instead of the elasticity tensor), $\omega$ is the angular frequency, $\Omega$ is a spectral set, $s(\omega)$ is the corresponding spectral density, $D$ denotes the protected zone (plane region), and $m$ is the magnitude of deflections in the protected zone. This problem resembles 
one that is usually solved at finding optimal parameters of shock absorbers [51-53].

4.2. Where Seismic Barriers Can Be Most Efficient? Simple observations reveal that different types of seismic barriers can be most efficient at soft soils especially subjected to liquefaction, when more traditional seismic protection measures can be inadequate. Indeed, by diminishing amplitude of seismic waves inside the protected zone, the considered barriers should improve stability of liquefied soils.

However, for such soils, a more complicated analysis of traveling waves involving Biot's theory of poroelasticity can be needed; see E. Detournay and Cheng [54]. It should also be mentioned that according to the genuine Biot's theory, all governing equations are linear, that ensures validity of the harmonic wave approach.

\section{Acknowledgment}

The authors thank the Russian Foundation for Fundamental Research (Grant no. 09-01-12063-ofi) for partial financial support.

\section{References}

[1] A. Takahashi, J. Takemura, and T. Shimodaira, "Seismic performance of reinforced earth wall with geogrid," in Proceedings of the 15th International Conference on Soil Mechanics and Geotechnical Engineering, pp. 1265-1268, Istanbul, Turkey, 2001.

[2] R. Motamed, K. Itoh, S. Hirose, A. Takahashi, and O. Kusakabe, "Evaluation of wave barriers on ground vibration reduction through numerical modeling in ABAQUS," in Proceedings of the SIMULIA Customer Conference 2009, pp. 402-441, London, UK, 2008.

[3] O. Kusakabe, J. Takemura, A. Takahashi, J. Izawa, and S. Shibayama, "Physical modeling of seismic responses of underground structures," in Proceedings of the 12th International Conference of International Association for Computer Methods and Advances in Geomechanics, pp. 1459-1474, Goa, India, 2008.

[4] S. V. Kuznetsov, "A new principle for protection from seismic waves," in Proceedings of the International Conference on Performance-Based Design in Earthquake Geotechnical Engineering, pp. 463-468, Tokyo, Japan, 2009.

[5] P. Chadwick and G. D. Smith, "Foundations of the theory of surface waves in anisotropic elastic materials," Advances in Applied Mechanics, vol. 17, pp. 303-376, 1977.

[6] A. E. H. Love, Some Problems of Geodynamics, Cambridge University Press, Cambridge, UK, 1911.

[7] K. Sobczyk, "Scattering of Reyleigh waves at a random boundary of an elastic body," Proceedings of Vibation Problems, vol. 7, no. 4, pp. 363-374, 1966.

[8] A. Maradudin and D. L. Mills, "Attenuation of Rayleigh surface waves by surface roughness," Applied Physics Letters, vol. 28, no. 10, pp. 573-575, 1976.

[9] A. A. Maradudin and J. Shen, "Multiple scattering of waves from random rough surfaces," Physical Review B, vol. 22, no. 9, pp. 4234-4240, 1980.
[10] F. Blondeau, "Fondations profondes-cours de mécanique des sols de l'E.N.P.C.," in Techniques de l'Ingérieur, chapitre C, p. 248, 1989.

[11] P. De Buhan, R. Mangiavacchi, R. Nova, G. Pellegrini, and J. Salencon, "Yield design of reinforced earth walls by a homogenization method," Géotechnique, vol. 39, no. 2, pp. 189-201, 1989.

[12] N. Abu-Hejleh, J. G. Zornberg, T. Wang, and J. Watcharamonthein, "Monitored displacements of unique geosyntheticreinforced soil bridge abutments," Geosynthetics International, vol. 9, no. 1, pp. 71-95, 2002.

[13] G. Eiksund, I. Hoff, and S. Perkins, "Cyclic triaxial tests on reinforced base course material," in Proceedings of the EuroGeo3, vol. 2, pp. 619-624, DGGT, Munich, Germany, 2004.

[14] V. Herle, "Long-term performance of reinforced soil structures," in Proceedings of the 13th Danube-Conference on Geotechnical Engineering, vol. 2, pp. 251-256, Slovenian Geotechnical Society, 2006.

[15] A. Bensoussan, J. L. Lions, and G. Papanicolaou, Asymptotic Analysis for Periodic Structures, North-Holland, Amsterdam, The Netherlands, 1978.

[16] E. Sanchez-Palencia, "Homogenization method for the study of composite media," in Asymptotic Analysis II, vol. 985 of Lecture Notes in Mathematics, pp. 192-214, Springer, 1983.

[17] J. C. Michel, H. Moulinec, and P. Suquet, "Effective properties of composite materials with periodic microstructure: a computational approach," Computer Methods in Applied Mechanics and Engineering, vol. 172, no. 1-4, pp. 109-143, 1999.

[18] A. Cecchi and N. L. Rizzi, "Heterogeneous elastic solids: a mixed homogenization-rigidification technique," International Journal of Solids and Structures, vol. 38, no. 1, pp. 29-36, 2001.

[19] J. W. Strutt, "On wave propagating along the plane surface of an elastic solid," Proceedings of the London Mathematical Society, vol. 17, pp. 4-11, 1885.

[20] T. C. Lim and G. W. Farnell, "Search for forbidden directions of elastic surface-wave propagation in anisotropic crystals," Journal of Applied Physics, vol. 39, no. 9, pp. 4319-4325, 1968.

[21] T. C. Lim and G. W. Farnell, "Character of pseudo surface waves on anisotropic crystals," Journal of the Acoustical Society of America, vol. 45, no. 4, pp. 845-851, 1969.

[22] G. W. Farnell, "Properties of elastic surface waves," Acoustical Physics, vol. 6, pp. 109-166, 1970.

[23] D. M. Barnett and J. Lothe, "Synthesis of the sextic and the integral formalism for dislocations, Green's functions, and surface waves in anisotropic elastic solids," Physica Norvegica, vol. 7, pp. 13-19, 1973.

[24] D. M. Barnett and J. Lothe, "Consideration of the existence of surface wave (Rayleigh wave) solutions in anisotropic elastic crystals," Journal of Physics F, vol. 4, no. 5, pp. 671-686, 1974.

[25] D. M. Barnett and J. Lothe, "An image force theorem for dislocations in anisotropic bicrystals," Journal of Physics F, vol. 4, no. 10, pp. 1618-1635, 1974.

[26] J. Lothe and D. M. Barnett, "On the existence of surface-wave solutions for anisotropic elastic half-spaces with free surface," Journal of Applied Physics, vol. 47, no. 2, pp. 428-433, 1976.

[27] P. Chadwick and D. A. Jarvis, "Surface waves in a prestressed elastic body," Proceedings of the Royal Society A, vol. 366, no. 1727, pp. 517-536, 1979.

[28] P. Chadwick and T. C. T. Ting, "On the structure and invariance of the Barnett-Lothe tensors," Quarterly of Applied Mathematics, vol. 45, pp. 419-427, 1987. 
[29] S. V. Kuznetsov, "Surface waves of non-Rayleigh type," Quarterly of Applied Mathematics, vol. 61, no. 3, pp. 575-582, 2003.

[30] R. Stoneley, "Elastic waves at the surface of separation of two solids," Proceedings of the Royal Society, vol. 106, pp. 416-428, 1924.

[31] K. Sezawa and K. Kanai, "The range of possible existence of Stoneley waves and some related problems," Bulletin of the Earthquake Research Institute, vol. 17, pp. 1-8, 1939.

[32] L. Cagniard, Reflexion et refraction des ondes seismique progressive, Ph.D. thesis, Gauthier-Villars \& Cie, Paris, France, 1939.

[33] J. G. Scholte, "The range of existence of Rayleigh and Stoneley waves," Monthly Notices of the Royal Astronomical Society, vol. 5, pp. 120-126, 1947.

[34] P. Chadwick and P. Borejko, "Existence and uniqueness of Stoneley waves," Geophysical Journal International, vol. 118, no. 2, pp. 279-284, 1994.

[35] P. R. Sengupta and S. Nath, "Surface waves in fiber-reinforced anisotropic elastic media," Sadhana, vol. 26, pp. 363-370, 2001.

[36] S. V. Kuznetsov, "Love waves in monoclinic media," Prikladnaya Matematika i Mekhanika, vol. 70, pp. 141-154, 2006 (Russian).

[37] S. V. Kuznetsov, "SH-waves in laminated plates," Quarterly of Applied Mathematics, vol. 64, no. 1, pp. 153-165, 2006.

[38] W. T. Thomson, "Transmission of elastic waves through a stratified solid medium," Journal of Applied Physics, vol. 21, no. 2, pp. 89-93, 1950.

[39] N. A. Haskell, "Dispersion of surface waves on multilayered media," Bulletin of the Seismological Society of America, vol. 43, no. 1, pp. 17-34, 1953.

[40] L. Knopoff, "A matrix method for elastic wave problems," Bulletin of the Seismological Society of America, vol. 54, no. 1, pp. 431-438, 1964.

[41] I. Djeran-Maigre and S. Kuznetsov, "Solitary SH waves in twolayered traction-free plates," Comptes Rendus, vol. 336, no. 1-2, pp. 102-107, 2008.

[42] D. C. Agnew, "History of seismology," International Handbook of Earthquake and Engineering Seismology, vol. 81, pp. 3-11, 2002.

[43] C. Braitenberg and M. Zadro, "Comparative analysis of the free oscillations generated by the Sumatra-Andaman Islands 2004 and the Chile 1960 earthquakes," Bulletin of the Seismological Society of America, vol. 97, supplement 1, pp. S6S17, 2007.

[44] H. Lamb, "On waves in an elastic plate," Proceedings of the Royal Society A, vol. 93, pp. 114-128, 1917.

[45] W. Lin and L. M. Keer, "A study of Lamb waves in anisotropic plates," Journal of the Acoustical Society of America, vol. 92, no. 2, pp. 888-894, 1992.

[46] T. C. T. Ting, Anisotropic Elasticity: Theory and Applications, Oxford University Press, New York, NY, USA, 1996.

[47] T. C. T. Ting, "An explicit secular equation for surface waves in an elastic material of general anisotropy," Quarterly Journal of Mechanics and Applied Mathematics, vol. 55, no. 2, pp. 297311, 2002.

[48] E. Buckingham, "On physically similar systems; illustrations of the use of dimensional equations," Physical Review, vol. 4, no. 4, pp. 345-376, 1914.

[49] E. Buckingham, “The principle of similitude," Nature, vol. 96, no. 2406, pp. 396-397, 1915.

[50] W. D. Curtis, J. D. Logan, and W. A. Parker, "Dimensional analysis and the pi theorem," Linear Algebra and Its Applications, vol. 47, pp. 117-126, 1982.
[51] J. P. Den Hartog, Mechanical Vibrations, Dover, New York, NY, USA, 1985.

[52] D. V. Balandin, N. N. Bolotnik, and W. D. Pilkey, "Optimal protection from impact and shock: theory and methods," Applied Mechanics Reviews, vol. 53, no. 9, pp. 237-264, 2000.

[53] D. V. Balandin, N. N. Bolotnik, W. D. Pilkey, S. V. Purtsezov, and C. G. Shaw, "Concept of a platform-based impact isolation system for protection of wheelchair occupants from injuries in vehicle crashes," Medical Engineering and Physics, vol. 30, no. 2, pp. 258-267, 2008.

[54] E. Detournay and A. H. D. Cheng, "Fundamentals of poroelasticity, Vol. II," in Comprehensive Rock Engineering: Principles, Practice \& Projects, Pergamon Press, New York, NY, USA, 1993. 

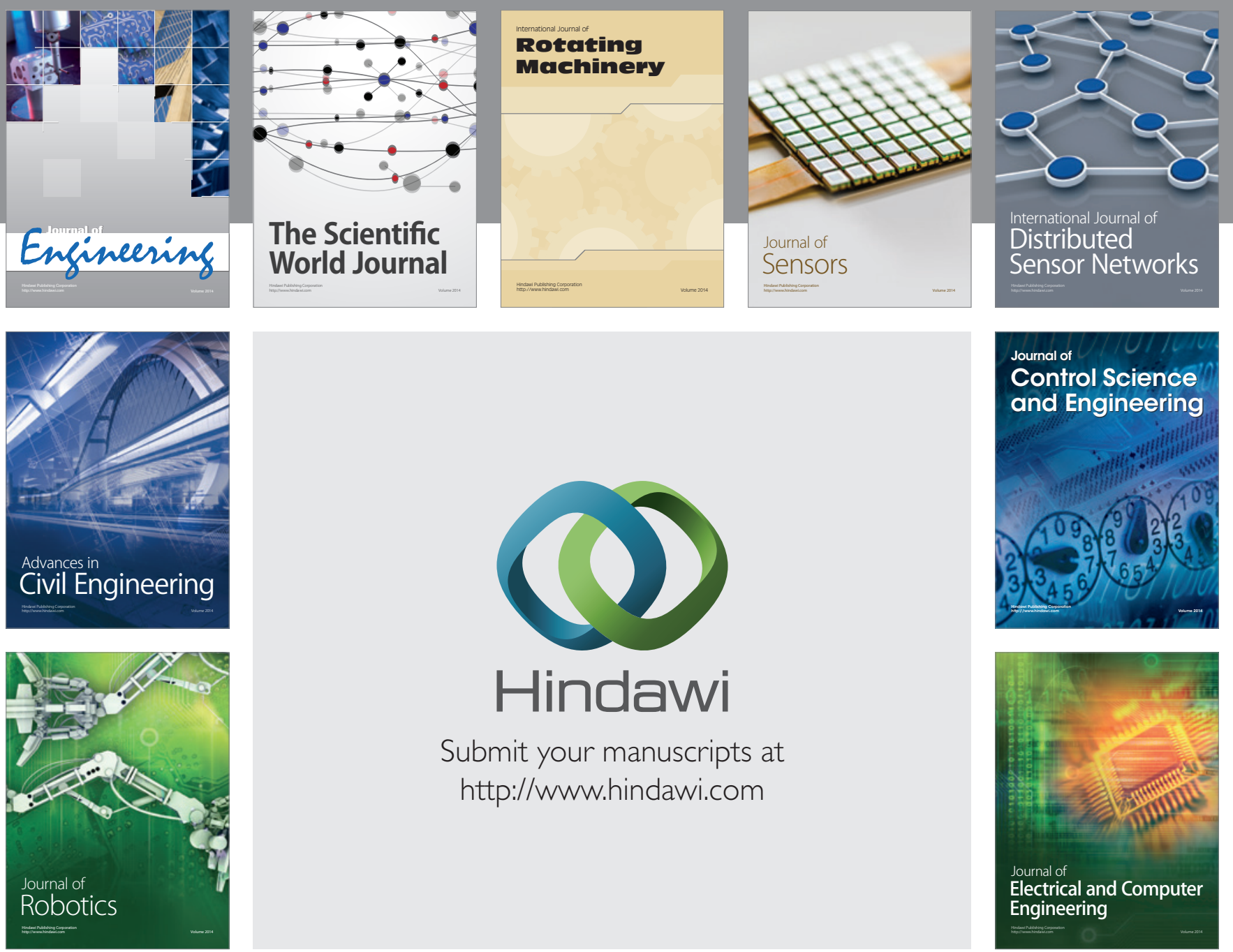

Submit your manuscripts at

http://www.hindawi.com
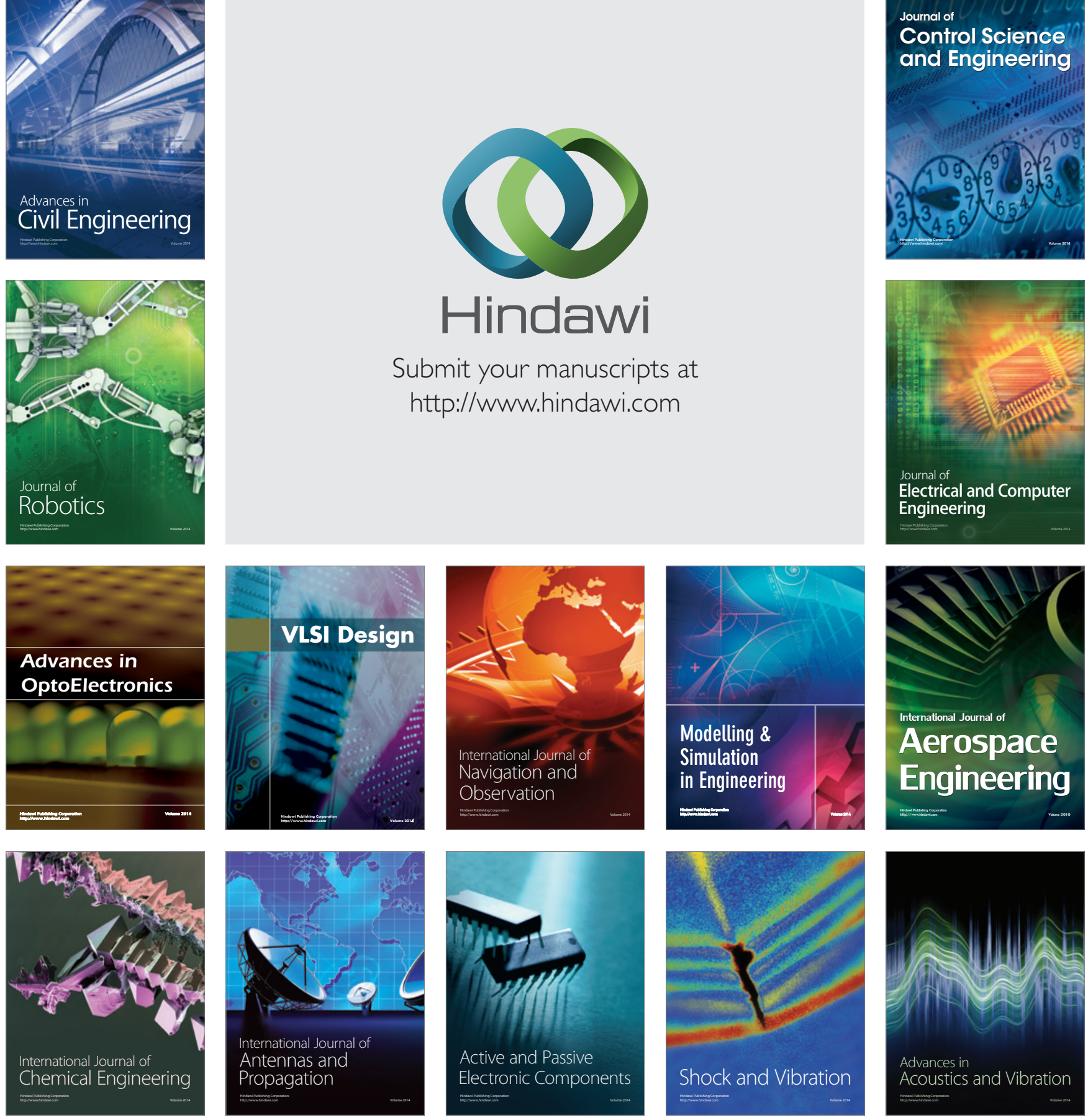